\title{
Downregulation of miR-95 in gastric cancer promotes EMT via regulation of Slug, thereby promoting migration and invasion
}

\author{
WEI ZHANG ${ }^{1}$, JING SUN $^{2}$, JINYAN CHEN $^{3}$, CAIYUN XU $^{3}$ and LIXIA ZHANG ${ }^{3}$ \\ Departments of ${ }^{1}$ Gastrointestinal Surgery and ${ }^{2}$ Emergency, Affiliated Hospital of Jining Medical University, Jining, \\ Shandong 272000; ${ }^{3}$ Department of Nuclear Medicine, Zhejiang Provincial Hospital of Traditional Chinese Medicine \\ (The First Affiliated Hospital of Zhejiang Chinese Medical University), Hangzhou, Zhejiang 310006, P.R. China
}

Received January 15, 2018; Accepted July 23, 2018

DOI: $10.3892 /$ or.2018.6911

\begin{abstract}
R-95 has been revealed to be aberrantly expressed in multiple types of cancer and to regulate tumor development. Moreover, miR-95 has been revealed to be downregulated in gastric cancer. However, the detailed function of miR-95 in gastric cancer has remained largely unknown. In the present study, we found that miR-95 was downregulated in 63 pairs of gastric cancer tissue samples and adjacent normal tissue samples, as well as gastric cancer cell lines. Additionally, the expression of miR-95 was associated with tumor size, tumor-node-metastasis (TNM) stage and lymph node metastasis. Various functional experiments, including Cell Counting Kit-8 (CCK-8), colony formation, wound healing and Transwell assays were used to explore the effect of miR-95 on cell proliferation and migration as well as invasion, respectively. Overexpression of miR-95 significantly suppressed cell proliferation, migration and invasion. Moreover, miR-95 inhibited EMT by directly binding the 3'-untranslated region (3'-UTR) of Slug. Therefore, miR-95 may be used as a novel therapeutic target for suppressing gastric cancer growth and metastasis.
\end{abstract}

\section{Introduction}

Gastric cancer has become the second highest leading cause of cancer-related deaths worldwide (1). Since the development of new diagnostic techniques as well as improvements in radical lymphadenectomy surgical approaches, the prognosis of gastric cancer patients has improved, but the incidence and mortality rates remain high. The 5-year overall survival rate is still at $20 \%(2-4)$, and the 5-year overall survival rate

Correspondence to: Dr Lixia Zhang, Department of Nuclear Medicine, Zhejiang Provincial Hospital of Traditional Chinese Medicine (The First Affiliated Hospital of Zhejiang Chinese Medical University), 54 Youdian Road, Hangzhou, Zhejiang 310006, P.R. China

E-mail: zhangman2233@yeah.net

Key words: miR-95, gastric cancer, proliferation, EMT, migration of patients with late stage is nearly 4\% (5). Therefore, it is urgent to identify potential predictive markers and effective molecular therapeutic targets of gastric cancer.

miRNAs (miRs), a class of small non-coding RNAs, have been reported to suppress the expression of multiple target genes by directly binding to a recognition sequence in the 3'-untranslated regions (3'-UTRs) of the mRNA of the target genes, causing mRNA degradation or translational repression (6-8). Increasing evidence has revealed that the expression of miRs is aberrant in various cancers, and the dysregulation of miRs plays crucial functions in the development and progression of cancers (9-12). Recently, miRs, including miR-93, miR-155 and miR-582 were revealed to promote or suppress gastric cancer proliferation and metastasis (13-15). Previous studies revealed that miR-95 was aberrantly expressed in multiple types of cancer and regulated tumor development (16-18). Chen et al revealed that miR-95 was downregulated in the GSRCC type of gastric cancer (19). However, the underlying mechanism of miR-95 has not yet been elucidated.

In the present study, it was demonstrated that miR-95 was downregulated in gastric cancer tissues and cell lines, consistent with a previously study (19). Moreover, the expression of miR-95 was significantly associated with tumor size, tumor-node-metastasis (TNM) stage and lymph node metastasis. Overexpression of miR-95 suppressed gastric cancer cell proliferation, migration and invasion. Additionally, miR-95 also regulated EMT in gastric cancer by directly inhibiting Slug. Collectively, our findings demonstrated that miR-95 is a tumor suppressor in gastric cancer.

\section{Materials and methods}

Patients and tissue samples. Patients admitted to the Affiliated Hospital of Jining Medical University between February 2012 and October 2016 were evaluated. These patients with gastric cancer included 41 males and 22 females aged between 32-86 years, with a mean age of 60.6 years. Clinical stages were classified according to the International Union against Cancer TNM classification system (20). The Research Ethics Committee of the Affiliated Hospital of Jining Medical University approved the present study (JN2017015), and all patients provided written informed consent. All tissue samples were stored at $-80^{\circ} \mathrm{C}$ before use. 
Cell lines and cell culture. Human gastric cancer cell lines, such as CTC-141 (Laboratory of Stem Cell Biology of Sichuan University, Sichuan, China) and MKN45 [American Type Culture Collection (ATCC) Manassas, VA, USA], as well as normal human gastric epithelium cell line GES-1 (Bogu Biotechnology, Shanghai, China) were maintained in the Dulbecco's modified Eagle's medium (DMEM) supplemented with $100 \mathrm{U} / \mathrm{ml}$ penicillin, and $100 \mu \mathrm{g} / \mathrm{ml}$ streptomycin as well as $10 \%$ fetal bovine serum (FBS) at $37^{\circ} \mathrm{C}$ with $5 \% \mathrm{CO}_{2}$.

Cell transfection. Mimic control and miR-95 mimics (miR-95 mimic), as well as inhibitor control and miR-95 inhibitors (miR-95 inhibitor) were purchased from Qiagen (Duesseldorf, Germany). Cells were transfected using Lipofectamine 3000 according to the manufacturer's instructions (Invitrogen; Thermo Fisher Scientific, Inc., Waltham, MA, USA). After transfection for $48 \mathrm{~h}$, the transfected cells were used for further experiments.

Quantitative real-time polymerase chain reaction ( $q R T-P C R)$. Total RNA was extracted from the gastric cancer tissue samples and cells using TRIzol reagent (Invitrogen; Thermo Fisher Scientific, Inc.) following the manufacturer's protocol and was quantified using NanoDrop 2000 (Thermo Fisher Scientific, Inc.). An RNA sample $(2 \mu \mathrm{g})$ was used to synthesize cDNA using RevertAid First Strand cDNA Synthesis Kit (Thermo Fisher Scientific,Inc.). A SYBR-Green (Roche Molecular Diagnostics, Pleasanton, CA, USA) was used to determine relative mRNA expression with an ABI PRISM 7500 Real-Time PCR System (Applied Biosystems; Thermo Fisher Scientific, Inc.) following the instructions of the manufacturer. Primers for miR-95 were purchased from GeneCopoeia Co. (Guangzhou, China). The cycling parameters were as follows: $95^{\circ} \mathrm{C}$ for $5 \mathrm{~min}$ and then 40 cycles of $95^{\circ} \mathrm{C}$ for $15 \mathrm{sec}$ and annealing/extension at $60^{\circ} \mathrm{C}$ for 1 min. E-cadherin: 5'-ACCTGGTTCAGATCAAATCC-3' (forward) and 5'-TCATTCTGATCGGTTACCGT-3' (reverse); N-cadherin: 5'-CAGAGTTTACTGCCATGACG-3' (forward) and 5'-AAAGTCGATTGGTTTGACCA-3' (reverse); vimentin: 5'-ATTGAGATTGCCACCTACAG-3' (forward) and 5'-ATCCAGATTAGTTTCCCTCAG-3' (reverse); Slug: 5'-AGATGCATATTCGGACCCACA-3' (forward) and 5'-CCTCATGTTTGTGCAGGAGAG-3' (reverse); GAPDH: 5'-GAGAAGTATGACAACAGCCTC-3' (forward) and 5'-ATGGACTGTGGTCATGAGTC-3' (reverse); miR-95: 5'-CTGGTGGAGGGATGGATG AA-3' (forward) and 5'-GGCCCGATCACAAACTCATC-3' (reverse); U6: 5'-CTCGCTTCGGCAGCACA-3' (forward) and 5'-AACGCTTCACGAATTTGCGT-3' (reverse) GAPDH mRNA or small nuclear RNA U6 were used as internal controls. The relative mRNA expression was calculated via the $2^{-\Delta \Delta C q}$ method (21).

Western blot analysis. Whole protein was isolated from the transfected cells using RIPA lysis buffer (Beyotime Institute of Biotechnology, Jiangsu, China), and protein concentrations were assessed using Pierce BCA Protein Assay kit (Pierce; Thermo Fisher Scientific, Inc.) following the manufacturer's instructions. Protein $\sim 45 \mu \mathrm{g}$ was separated via $10 \%$ SDS-PAGE. After being transferred to nitrocellulose
Table I. Clinicopathological variables in 63 gastric cancer patients.

\begin{tabular}{|c|c|c|c|c|}
\hline \multirow[b]{2}{*}{ Variables } & \multirow[b]{2}{*}{$\begin{array}{c}\text { No. } \\
(\mathrm{n}=63)\end{array}$} & \multicolumn{2}{|c|}{$\begin{array}{l}\text { miR-95 } \\
\text { expression }\end{array}$} & \multirow[b]{2}{*}{ P-value } \\
\hline & & $\begin{array}{l}\text { Low } \\
(n=42)\end{array}$ & $\begin{array}{l}\text { High } \\
(n=21)\end{array}$ & \\
\hline Age, years & & & & 0.285 \\
\hline$<60$ & 33 & 20 & 13 & \\
\hline$\geq 60$ & 30 & 22 & 8 & \\
\hline Sex & & & & 0.135 \\
\hline Male & 41 & 30 & 11 & \\
\hline Female & 22 & 12 & 10 & \\
\hline Tumor size (diameter) (cm) & & & & 0.031 \\
\hline Small $(\leq 3)$ & 27 & 14 & 13 & \\
\hline Large $(\geq 3)$ & 36 & 28 & 8 & \\
\hline TNM stage & & & & 0.020 \\
\hline I-II & 29 & 15 & 14 & \\
\hline III-IV & 34 & 27 & 7 & \\
\hline Lymph node metastasis & & & & 0.021 \\
\hline No & 32 & 17 & 15 & \\
\hline Yes & 31 & 25 & 6 & \\
\hline
\end{tabular}

TNM, tumor node metastasis.

filter membranes (EMD Millipore, Bedford, MA, USA), the membranes were blocked with $5 \%$ skimmed milk at room temperature for $1 \mathrm{~h}$. Subsequently, the membranes were incubated with indicated primary antibodies at $4{ }^{\circ} \mathrm{C}$ overnight. After being washed with phosphate-buffered saline Tween-20 (PBST) three times at room temperature, the membranes were incubated with HRP-conjugated secondary antibodies at room temperature for $1 \mathrm{~h}$ and washed with PBST three times at room temperature. Finally, the blots were visualized by ECL kit (Pierce; Thermo Fisher Scientific, Inc.). Each independent experiment was performed three times. The anbodies as follow: EMT kit (1:1,000; cat. no. 9782; Cell Signaling Technology, Danvers, MA, USA), $\beta$-actin (1:2,000; cat. no. ab6276; Abcam, Cambridge, UK), goat anti-rabbit (HRP) (1:5,000; cat. no. ab205718; Abcam), goat anti-mouse (HRP) (1:3,000; cat. no. ab205719, Abcam). The densitometry of blots was determined using ImageJ software (version 4.1; National Institutes of Health, Bethesda, MD, USA).

Cell Counting Kit-8 (CCK-8) assay. CCK-8 assay was used to detect the effect of miR-95 on cell proliferation. In brief, 3,000 transfected CTC-141 or MKN45 cells with $200 \mu 1$ media were seeded in 96-well plates. After transfection for $48 \mathrm{~h}$, $20 \mu \mathrm{l} \mathrm{CCK}-8$ solution (Beyotime Institute of Biotechnology) was added to the culture medium at $0,24,48$ and $72 \mathrm{~h}$, and incubated for $30 \mathrm{~min}$ at $37^{\circ} \mathrm{C}$. The absorbance of $450 \mathrm{~nm}$ was measured using a microplate reader (Bio-Rad Laboratories, Inc., Hercules, CA, USA). Each independent experiment was performed three times. 
A

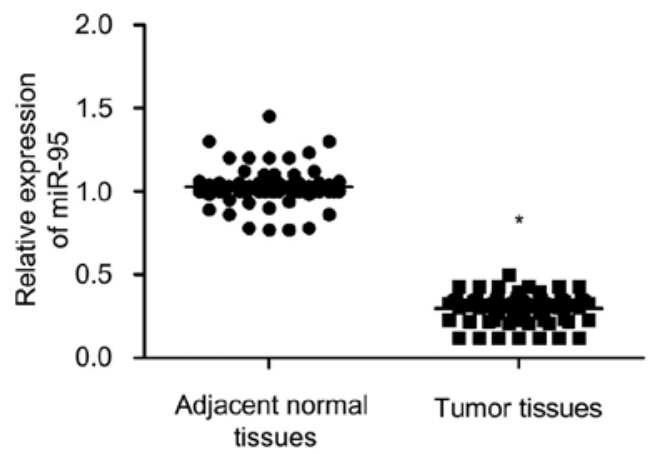

B

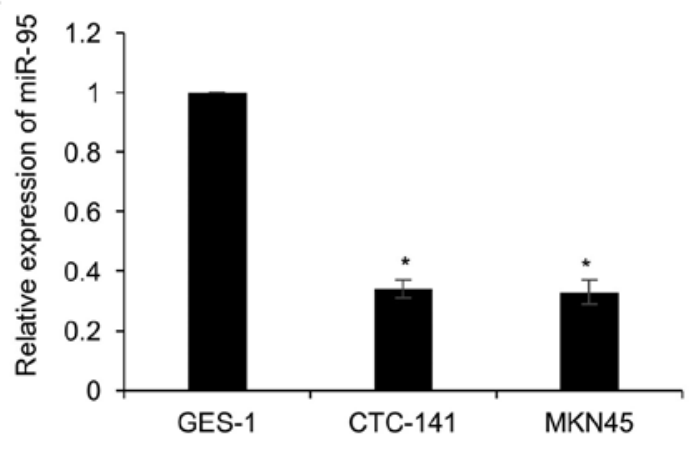

Figure 1. miR-95 is aberrantly downregulated in gastric cancer tissues as well as cell lines, and associated with poor tumor progression. (A) qRT-PCR result of the expression of miR-95 in 63 pairs of gastric cancer tissues and adjacent normal tissues. Tumor tissues vs. adjacent normal tissues, ${ }^{*} \mathrm{P}<0.05$. (B) qRT-PCR result of the expression of miR-95 in gastric cancer cell lines, such as CTC-141 and MKN45. GES-1 was used as a control. CTC-141 or MKN45 vs. GES-1, ${ }^{*} \mathrm{P}<0.05$.

Wound healing assay. A wound healing assay was used to determine the effect of miR-95 on cell migration. Briefly, $5 \times 10^{5}$ transfected CTC-141 or MKN45 cells were seeded in 6-well plates. When cell density was almost $90-100 \%$, a linear wound was generated using a $20-\mu 1$ pipette tip, and the detached cells were washed with PBS three times. The distance of wound healing was assessed at 0 and $24 \mathrm{~h}$ with a light microscope. Each independent experiment was performed three times.

Transwell invasion assay. A Transwell assay was used to determine the effect of miR-95 on cell invasion. In brief, $80 \mu 1$ Matrigel was coated on the Transwell chambers (BD Biosciences, Bedford, MA, USA) and maintained at $37^{\circ} \mathrm{C}$ for $30 \mathrm{~min}$. Approximately $4 \times 10^{4}$ transfected CTC-141 or MKN45 cells in $400 \mu \mathrm{l}$ serum-free medium were placed in the upper chamber in 24-well culture plates, and $500 \mu \mathrm{l}$ RPMI1640 medium containing $10 \%$ FBS was added to the lower chamber. Cells were maintained at $37^{\circ} \mathrm{C}$ with $5 \% \mathrm{CO}_{2}$ for $16 \mathrm{~h}$. Subsequently, the cells were stained with $0.5 \%$ crystal violet at room temperature for $10 \mathrm{~min}$. The cells on the surface of the upper membranes were removed by cotton swab, and the number of invading cells was counted under a light microscope. Each independent experiment was performed three times.

EMT induction. When cell density was almost $60 \%$, cells were cultured with serum-free media overnight. Recombinant human TGF- $\beta 1$ (10 ng/ml) was added into media for $72 \mathrm{~h}$.

Luciferase reporter assay. TargetScan Human version 7.0 (www.targetscan.org) predicted that Slug was a potential target of miR-95. The 3'-UTR of Slug was cloned into the pGL3 luciferase vector (Invitrogen; Thermo Fisher Scientific, Inc.). For the luciferase assay, CTC-141 and MKN-45 cells were co-transfected with Renilla, pGL3-Slug 3'-UTR or and miR-95 mimics or miR-95 inhibitor. After transfection for $24 \mathrm{~h}$, a luciferase reporter assay was performed using a DualLuciferase Reporter Assay kit according to the manufacturer's instructions (Promega Corp., Madison, Wisconsin, USA). The Renilla luciferase activity was used to normalize firefly luciferase activity. Each independent experiment was performed three times.
Statistical analysis. All data were analyzed using SPSS 18.0 statistical software (SPSS, Inc., Chicago, IL, USA) and presented as the mean \pm SD. A Student's t-test or one-way ANOVA followed by Tukey's were used to analyze the differences between groups. $\mathrm{P}<0.05$ was considered to indicate a statistically significant difference.

\section{Results}

miR-95 is aberrantly downregulated in gastric cancer tissues as well as cell lines, and associated with poor tumor progression. To investigate the functions of miR-95 in gastric cancer, we first detected the expression of miR-95 in gastric cancer tissues. We performed qRT-PCR to analyze the miR-95 expression levels in 63 gastric tumor tissue samples and adjacent normal tissues samples. As shown in Fig. 1A, our findings revealed that miR-95 expression was significantly downregulated in gastric cancer tissues compared with adjacent normal tissues (Fig. 1A). In addition, we detected the expression of miR-95 in gastric cancer cell lines, including CTC-141 and MKN45. GES-1 was used as a control. The expression of miR-95 was lower in CTC-141 and MKN45 cells compared to GES-1 (Fig. 1B). Subsequently, clinicopathological analyses of 63 gastric cancer patients demonstrated that the expression of miR-95 was closely associated with tumor size, lymph node metastasis as well as TNM stage (Table I).

miR-95 suppresses gastric cancer cell proliferation. To explore the function of $\mathrm{miR}-95$ in gastric cancer, we first overexpressed or knocked down miR-95 in CTC-141 and MKN45 cells with miR-95 mimics or miR-95 inhibitor. The expression of miR-95 was determined by qRT-PCR (Fig. 2A). As shown in Table I, since the expression of miR-95 was closely associated with tumor size, we assumed that miR-95 may regulate cell proliferation. To verify our hypothesis, CCK-8 and colony formation assays were performed. The results of the CCK-8 assay revealed that compared with that of the mimic control or inhibitor control groups, the proliferation of CTC-141 and MKN45 cells in the miR-95-mimic group were significantly decreased and were clearly increased in the miR-95-inhibitor group (Fig. 2B). The colony formation assay also confirmed that 
A

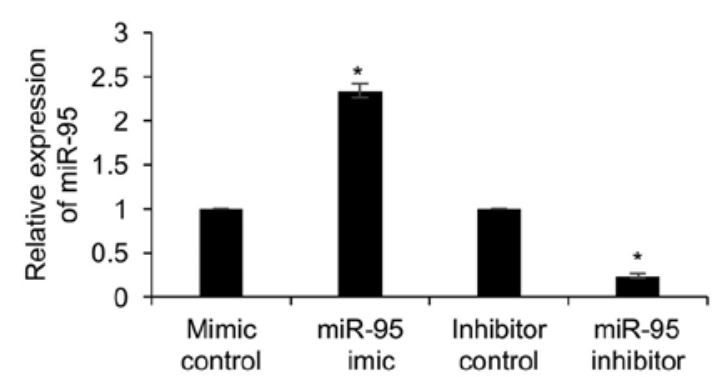

B

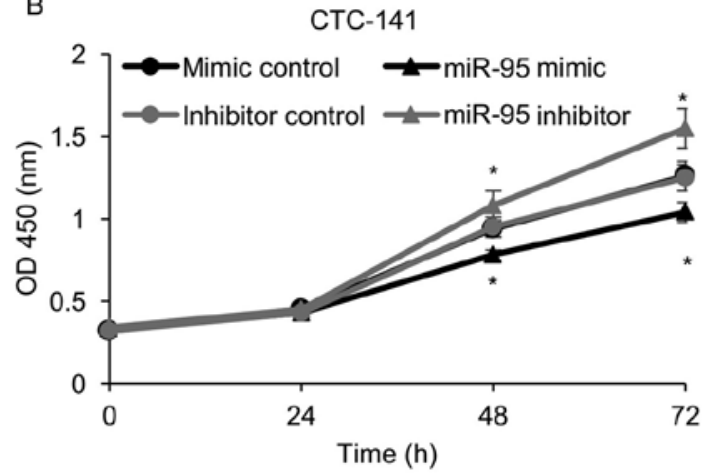

C

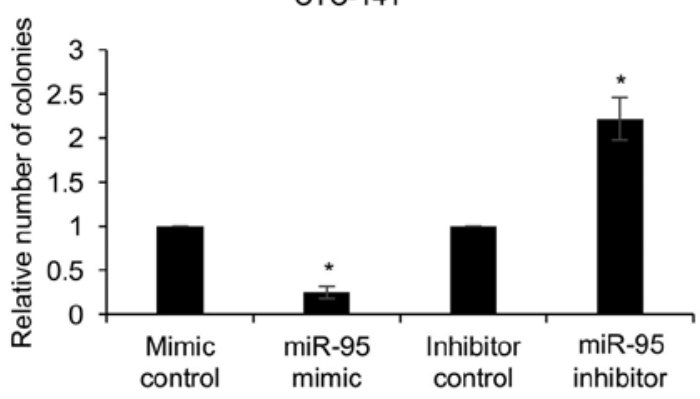

MKN45

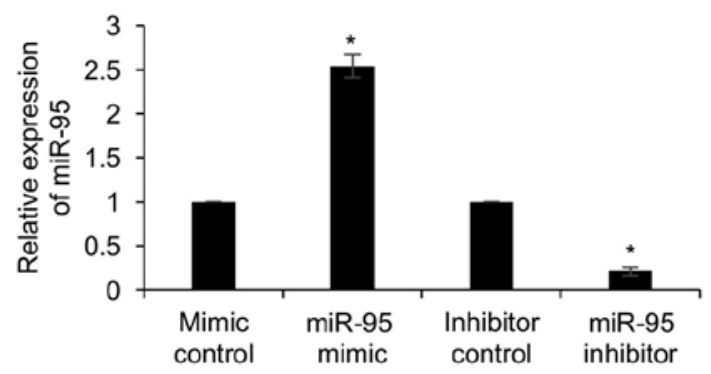

MKN45

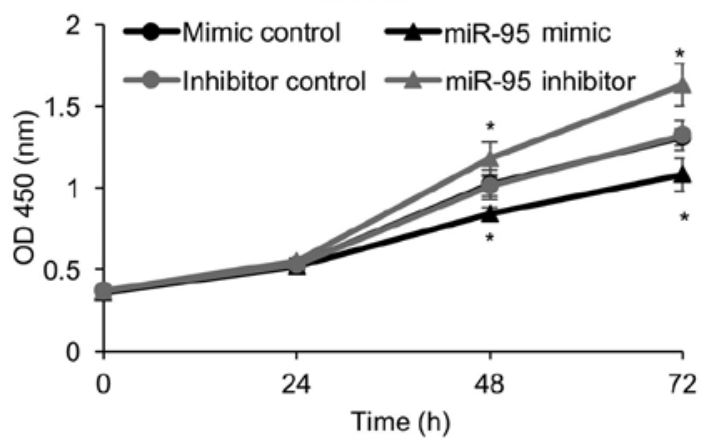

MKN45

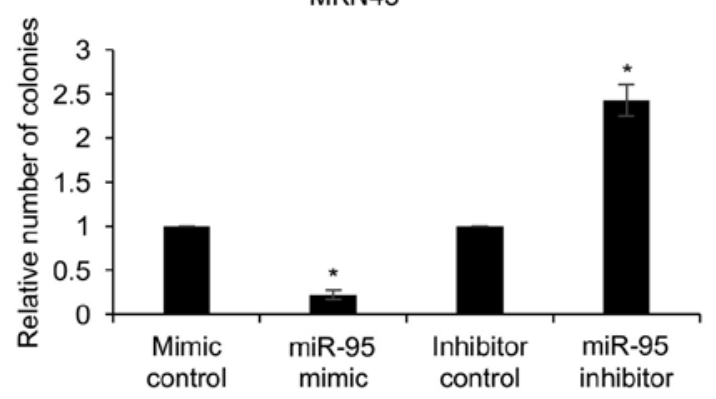

Figure 2. miR-95 suppresses gastric cancer cell proliferation. (A) miR-95 mimics or inhibitors significantly increased or decreased the expression of miR-95 in CTC-141 and MKN45 cells, respectively. miR-95 mimic vs. mimic control, miR-95 inhibitor vs. inhibitor control, ${ }^{*} \mathrm{P}<0.05$. (B) Representative graphs of the CCK-8 assay revealing CTC-141 and MKN45 cells transfected with miR-95 mimics or inhibitors. miR-95 mimic vs. mimic control, miR-95 inhibitor vs. inhibitor control, ${ }^{*} \mathrm{P}<0.05$. (C) Colony formation assay of CTC-141 and MKN45 cells transfected with miR-95 mimics or inhibitors. miR-95 mimic vs. mimic control, miR-95 inhibitor vs. inhibitor control, "P<0.05. CCK-8, Cell Counting Kit-8.

ectopic expression of miR-95 led to a decrease in the number of colonies, and an inhibition of miR-95 led to an increase in the number of colonies (Fig. 2C). These results revealed that miR-95 suppressed gastric cancer cell proliferation.

Downregulation of miR-95 promotes gastric cancer cell migration and invasion. Our experiments revealed that miR-95 expression was negatively associated with lymph node metastasis. To determine whether miR-95 regulated migration and invasion in gastric cancer cells, we performed wound healing and Transwell assays. The results of the wound healing assays revealed that ectopic expression of miR-95 significantly decreased the distance of cell migration (Fig. 3A). In contrast, downregulation of miR-95 significantly increased the distance of cell migration (Fig. 3A). Similar results were observed in the Transwell assay, revealing that the ectopic expression of miR-95 resulted in less invading cells than that in the mimic control group (Fig. 3B). In contrast, the downregulation of miR-95 resulted in more invading cells than those in the inhibitor control group (Fig. 3B). Since MMP9 is an invasionrelated factor, we next determined whether miR-95 regulated MPP9 secretion using an ELISA assay, which revealed that the secretion of MMP9 was decreased when cells were transfected with miR-95 mimics, and increased when cells were transfected with the miR-95 inhibitor (Fig. 3C). Our experiments revealed that downregulation of miR-95 promoted gastric cancer cell migration and invasion.

miR-95 inhibits the TGF- $\beta 1$-induced EMT process of gastric cancer cells. To further decipher the detailed mechanisms of miR-95 in gastric cancer metastasis, we aimed to explore the effects of miR-95 on EMT, which was recognized as a main cause for cell migration and invasion. CTC-141 and MKN45 cells were transfected with miR-95 mimics and mimic control. After inducing $10 \mathrm{ng} / \mathrm{ml}$ of TGF- $\beta 1$, the expression of EMT-associated proteins was determined by RT-qPCR and western blotting, respectively. The results revealed that ectopic expression of miR-95 led to an increased expression 

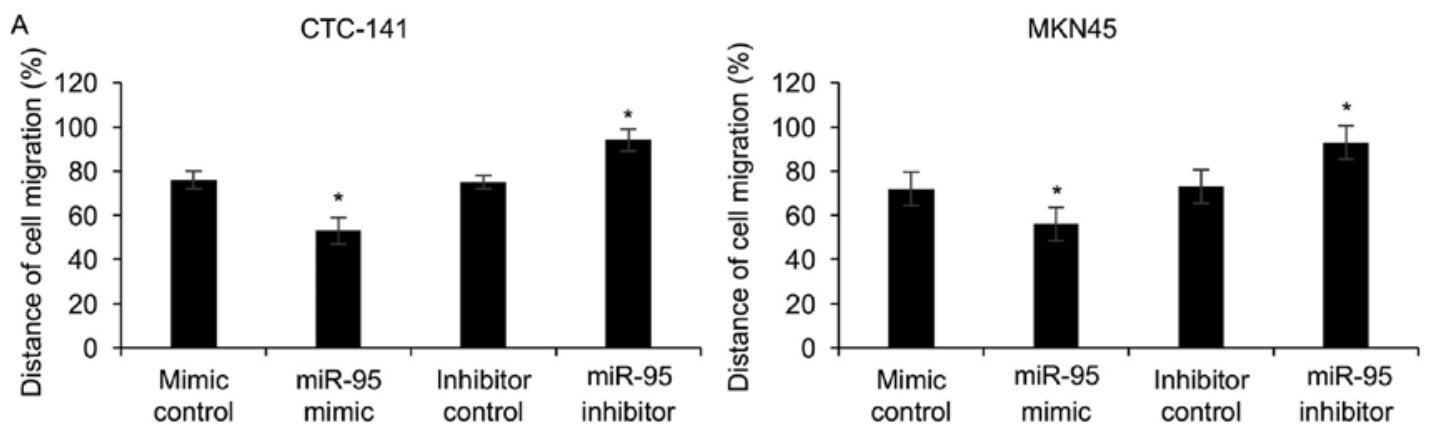

B
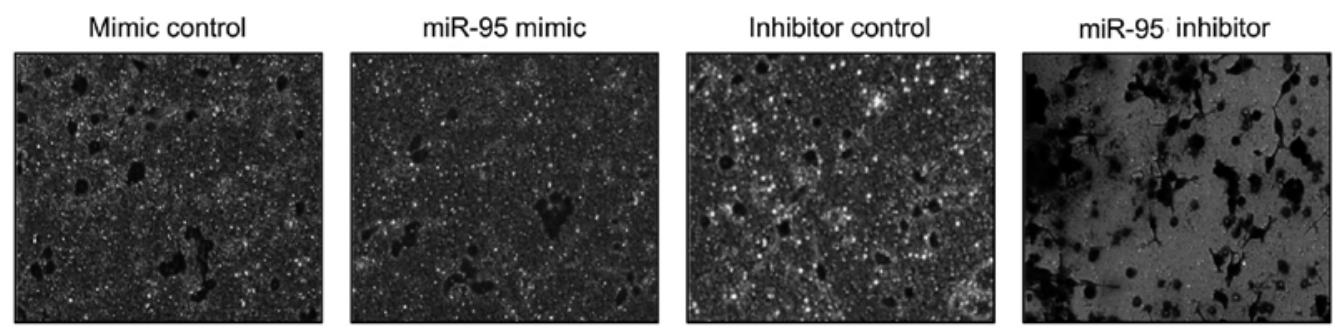

CTC-141
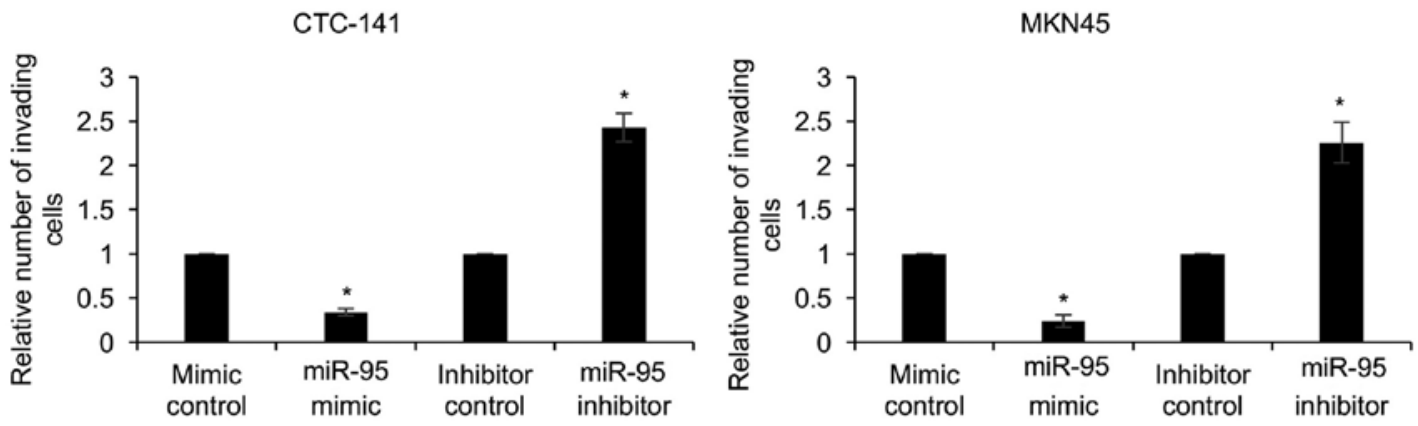

C

CTC-141

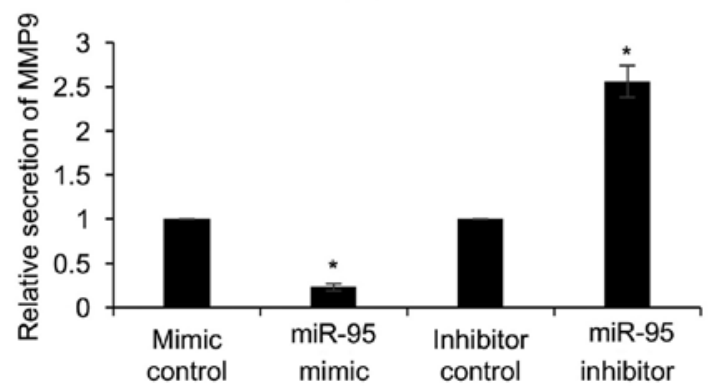

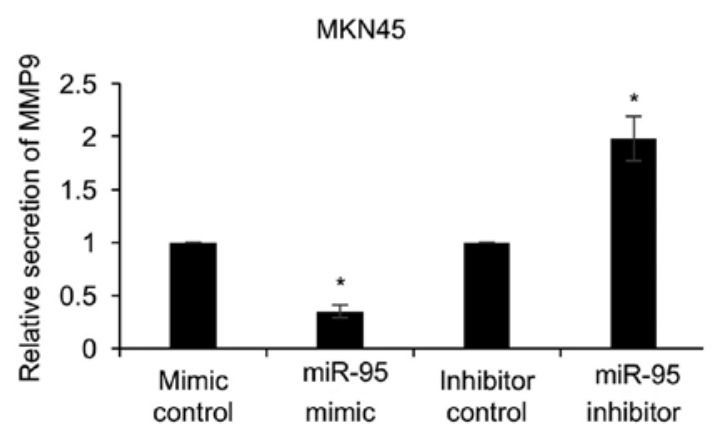

Figure 3. Downregulation of miR-95 promotes gastric cancer cell migration and invasion. (A) A wound healing assay of CTC-141 and MKN45 cells transfected with miR-95 mimics or inhibitors. miR-95 mimic vs. mimic control, miR-95 inhibitor vs. inhibitor control, "P<0.05. (B) A Transwell assay of CTC-141 and MKN45 cells transfected with miR-95 mimics or inhibitors. miR-95 mimic vs. mimic control, miR-95 inhibitor vs. inhibitor control, ${ }^{\text {"P }}<0.05$. (C) Levels of MMP9 proteins in gastric cancer cells assessed by ELISA analysis. miR-95 mimic vs. mimic control, miR-95 inhibitor vs. inhibitor control, ${ }^{*} \mathrm{P}<0.05$.

of E-cadherin both at the mRNA level and protein level, and a decreased expression of $\mathrm{N}$-cadherin and vimentin both at the mRNA level and protein level (Fig. 4A and B). A suppression of miR-95 reversed these results (Fig. 4C and D). The mRNA and protein levels of E-cadherin were decreased, and the levels of $\mathrm{N}$-cadherin as well as vimentin were increased (Fig. 4C and D). Overall, the results implied that miR-95 inhibits the TGF- $\beta 1$-induced EMT process of gastric cancer cells.
Slug is a target of miR-95 in gastric cancer cells. miRNAs have been found to participate in multiple physiological and pathological processes by regulating gene expression. To further decipher the detailed mechanism of miR-95 on EMT, we searched for potential targets of miR-95 by bioinformatics search using TargetScan (www.targetscan.org). We found that Slug, a key transcription factor of EMT, was a direct target of miR-95. To verify whether slug was regulated by miR-95, 
A

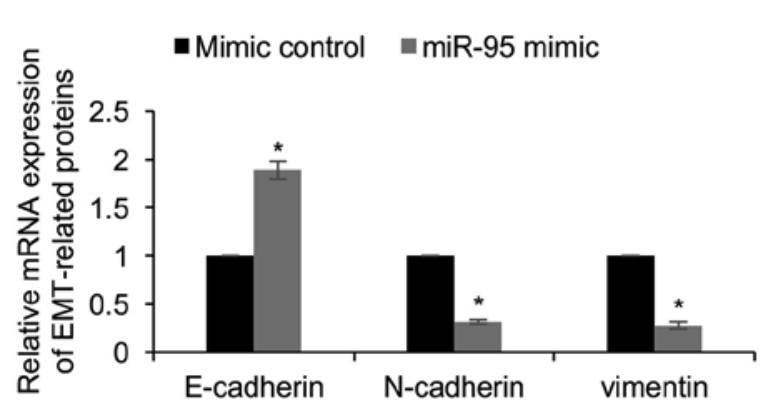

B

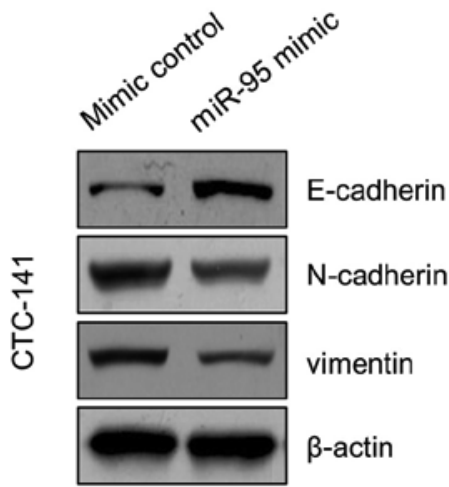

C

CTC-141

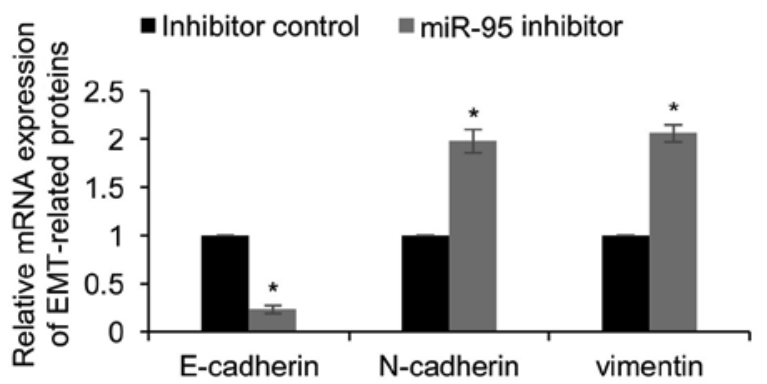

D

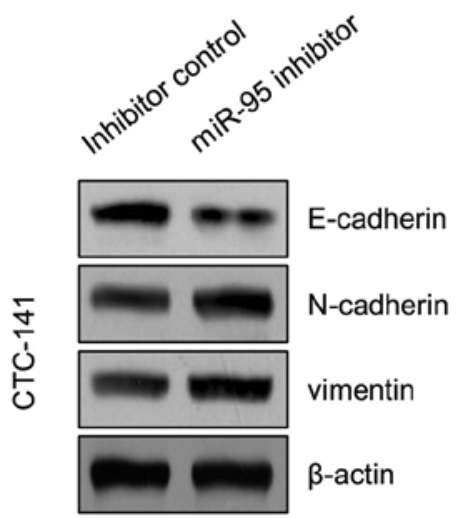

MKN45
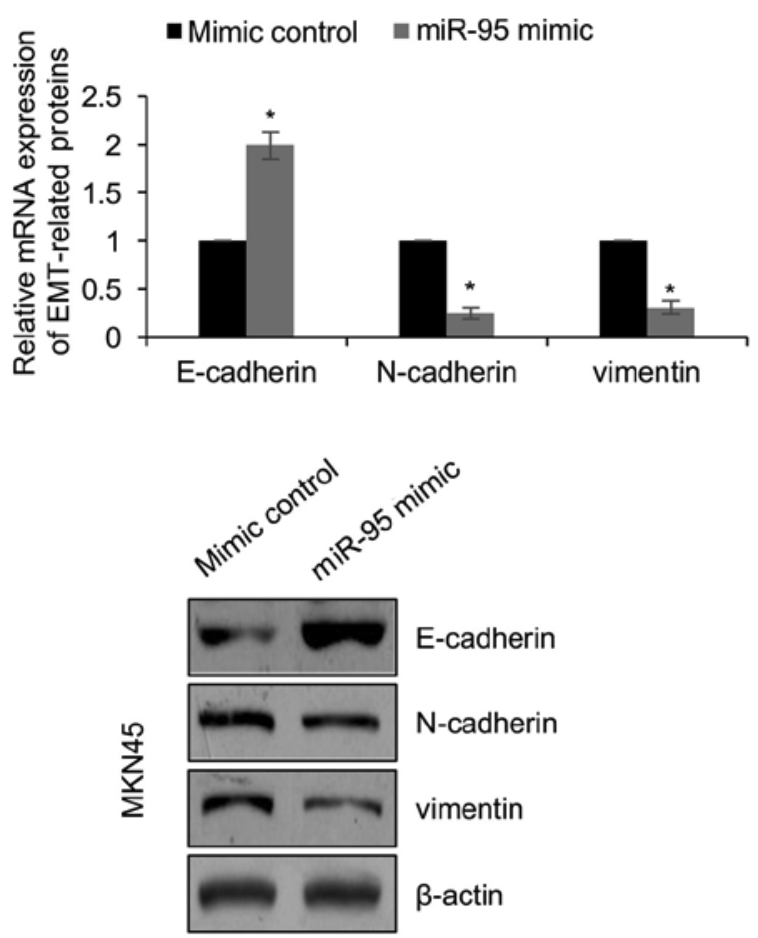

MKN45
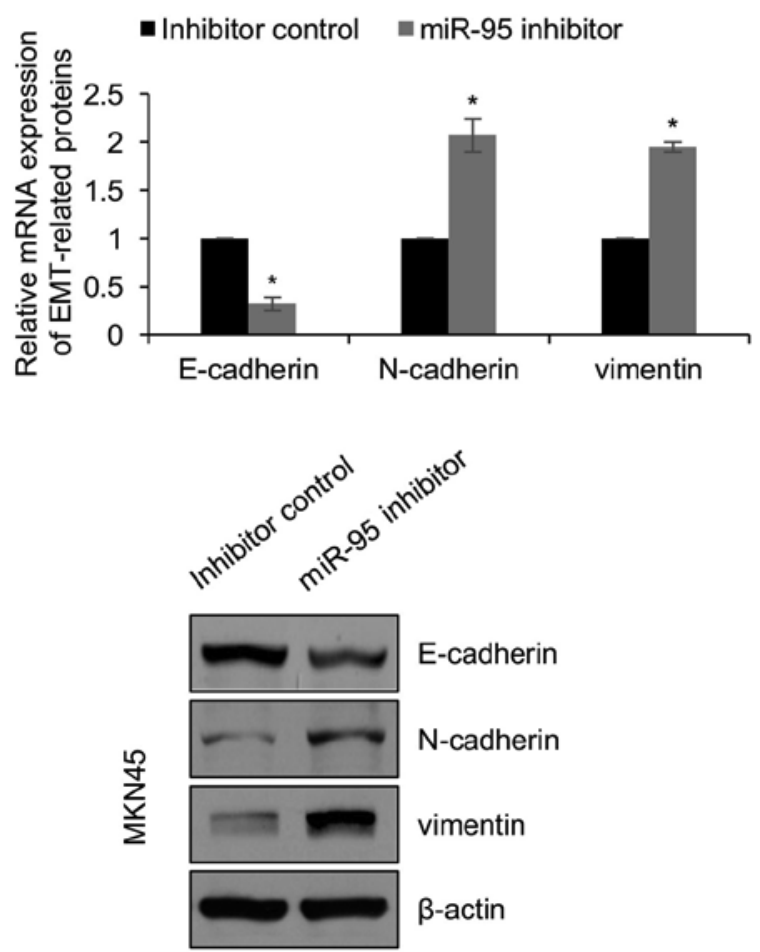

Figure 4. miR-95 inhibits the TGF- $\beta 1$-induced EMT process of gastric cancer cells. (A and B) miR-95 was overexpressed in CTC-141 and MKN45 cells. qRT-PCR and western blot analysis of E-cadherin, N-cadherin and vimentin in TGF- $\beta 1$-treated CTC-141 and MKN45 cells. miR-95 mimic vs. mimic control, "P<0.05. (C and D) miR-95 was knocked down in CTC-141 and MKN45 cells. qRT-PCR and western blot analysis of E-cadherin, N-cadherin and vimentin in TGF- $\beta 1$-treated CTC-141 and MKN45 cell lines. miR-95 inhibitor vs. inhibitor control, ${ }^{*} \mathrm{P}<0.05$.

we overexpressed or knocked down miR-95 in CTC-141 and MKN45 and detected the expression of Slug. As shown in Fig. 5A, we found that Slug expression was decreased in
CTC-141 and MKN45 cells treated with miR-95 mimics, while Slug expression was increased in CTC-141 and MKN45 cells treated with miR-95 inhibitors (Fig. 5A and B). To 
A
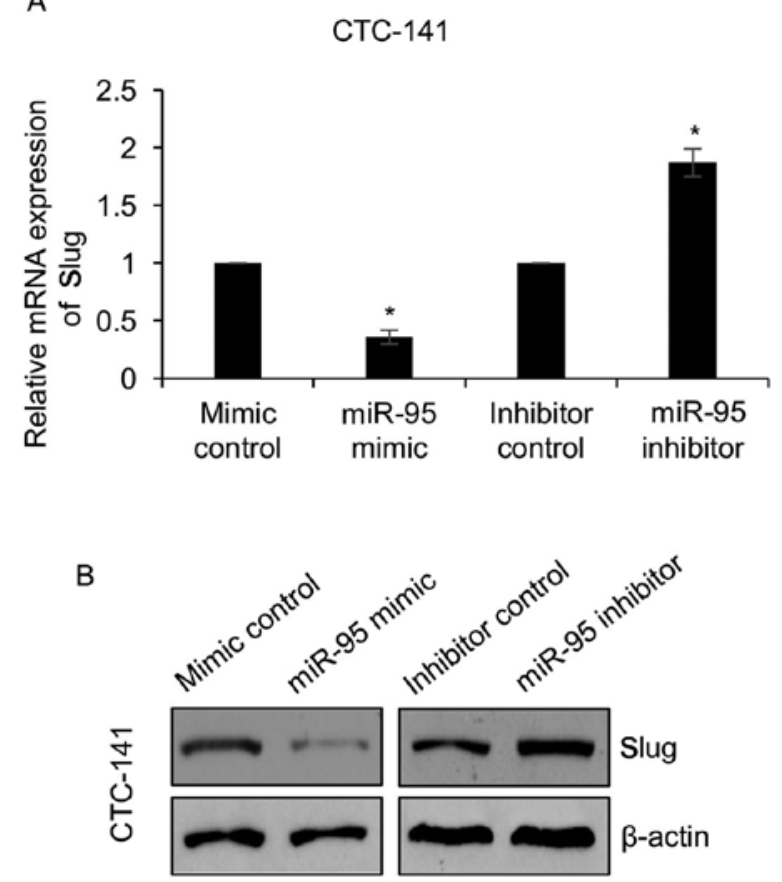

C

CTC-141

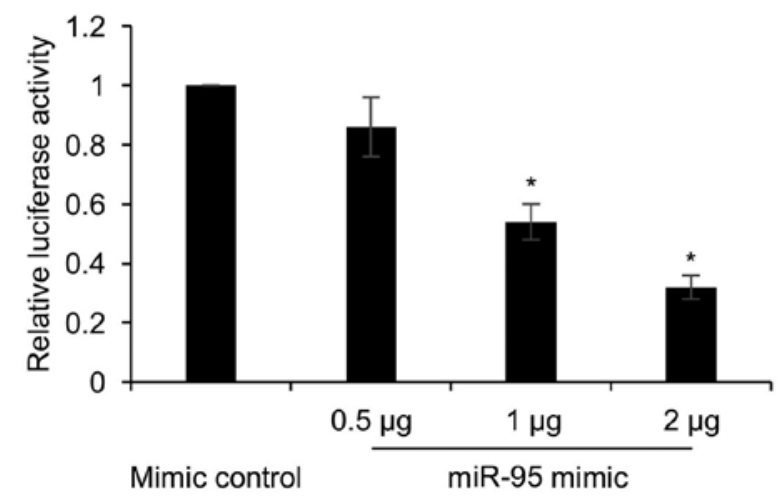

MKN45
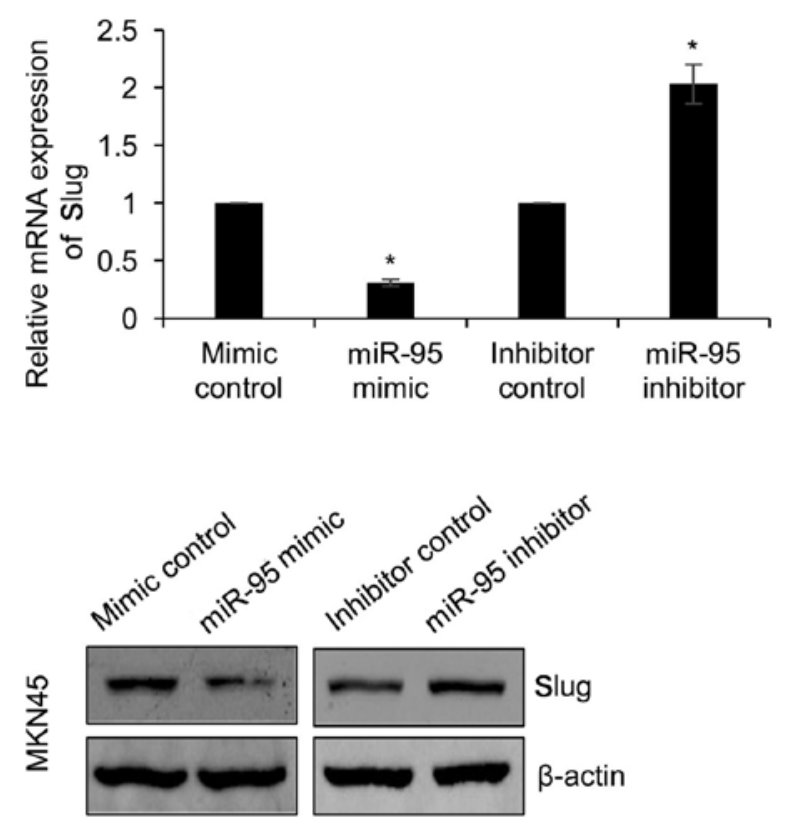

MKN45

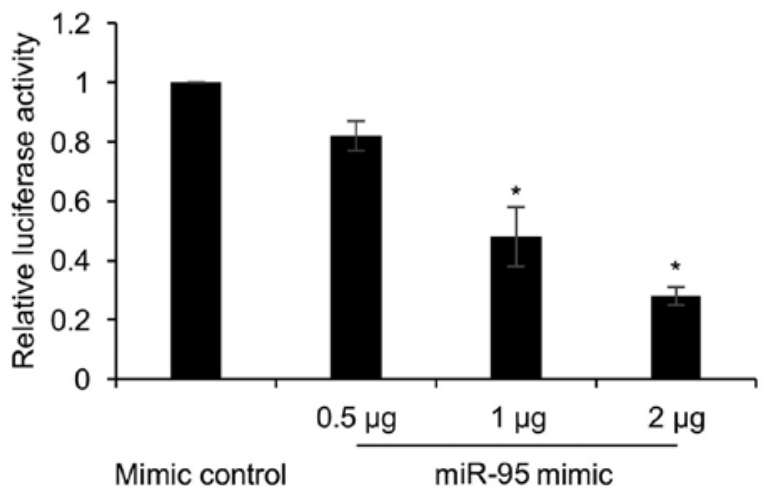

Figure 5. Slug is a target of miR-95 in gastric cancer cells. (A and B) miR-95 was overexpressed or knocked down in CTC-141 and MKN45 cells. qRT-PCR and western blot analysis of Slug. miR-95 mimic vs. mimic control, miR-95 inhibitor vs. inhibitor control, *P<0.05. (C) Upregulation of miR-95 significantly inhibited the luciferase activity of the 3'-UTR of Slug. miR-95 mimic vs. mimic control, ${ }^{*} \mathrm{P}<0.05$.

examine whether miR-95 directly interacted with the 3'-UTR of Slug, we performed a luciferase reporter assay. The luciferase activity was significantly decreased when cells were co-transfected with Slug 3'-UTR and either miR-95 mimics or mimic control (Fig. 5C). These findings indicated that Slug is a target of miR-95 in gastric cancer.

\section{Discussion}

miR-95 has been reported to facilitate cell proliferation in nonsmall cell lung cancer (NSCLC) and colorectal carcinoma, suggesting that miR-95 acts as an oncogenic miRNA $(17,22)$. Paradoxically, Chen et al revealed that miR-95 was downregulated in the GSRCC type of gastric cancer (19). Moreover, miR-95 was also revealed to regulate chemoresistance and radioresistance in NSCLC (22). A previous study also revealed that miR-95 plays a key function in the anticancer activity of Brucein D which is in contrast to its proliferative effects (23). Several studies have indicated that miRNAs are new therapeutic targets for multiple diseases, such as cancers. In colorectal cancer (CRC), miR-95 was revealed to be overexpressed in the serum sample of CRC patients (24), suggesting that miR-95 may be a potential molecular target for cancer diagnosis.

In this study, we found miR-95 was downregulated in gastric cancer tissues and cell lines. Additionally, the expression of miR-95 was significantly associated with tumor size, TNM stage and lymph node metastasis. Furthermore, we found that miR-95 suppressed proliferation, migration and invasion of gastric cancer cells.

Metastasis is the main reason for treatment failure in cancer and results in $>90 \%$ of cancer-related deaths (25-27). EMT is 
a complex process which is associated with the progression of metastasis, and improves the migration as well as invasive abilities of cancer cells. Various studies have indicated that miRNAs are involved in this process $(25,28)$. In the present study, we found that miR-95 suppressed EMT in gastric cancer cells. Notably, we found that Slug, a key transcription factor of EMT, was a target of miR-95. Therefore, we demonstrated that miR-95 suppressed cell migration and invasion through regulation of EMT in gastric cancer cells.

However, there are still some limitations in the present study. The upstream of miR-95 and detailed mechanism of miR-95 on cellular proliferation in gastric cancer is still unknown. Moreover, the function of miR-95 in vivo warrants further investigation.

Collectively, our findings revealed the important role of miR-95 in regulating EMT of gastric cancer. miR-95 suppressed Slug expression to regulate the progression of EMT, thereby inhibiting migration and invasion in gastric cancer. In addition, aberrant expression of miR-95 was closely associated with tumor size, lymph node metastasis as well as TNM stage, suggesting that miR-95 may play a key role in gastric cancer development. Our findings revealed miR-95 as a novel molecular therapeutic target of gastric cancer.

\section{Acknowledgements}

Not applicable.

\section{Funding}

No funding was received.

\section{Availability of data and materials}

The datasets used during the present study are available from the corresponding author upon reasonable request.

\section{Authors' contributions}

WZ and LZ conceived and designed the study. WZ, JS, CX and JC performed the experiments. WZ and CX wrote the paper. WZ and LZ reviewed and edited the manuscript. All authors read and approved the manuscript and agree to be accountable for all aspects of the research in ensuring that the accuracy or integrity of any part of the work are appropriately investigated and resolved.

\section{Ethics approval and consent to participate}

The Research Ethics Committee of the Affiliated Hospital of Jining Medical University, Jining, China approved the present study. All patients provided written informed consent.

\section{Patient consent for publication}

Not applicable.

\section{Competing interests}

The authors declare that they have no competing interests.

\section{References}

1. Torre LA, Bray F, Siegel RL, Ferlay J, Lortet-Tieulent J and Jemal A: Global cancer statistics, 2012. CA Cancer J Clin 65: 87-108, 2015

2. Shen L, Shan YS, Hu HM, Price TJ, Sirohi B, Yeh KH, Yang YH, Sano T, Yang HK, Zhang X, et al: Management of gastric cancer in Asia: Resource-stratified guidelines. Lancet Oncol 14: e535-e547, 2013.

3. Miller KD, Siegel RL, Lin CC, Mariotto AB, Kramer JL, Rowland JH, Stein KD, Alteri R and Jemal A: Cancer treatment and survivorship statistics, 2016. CA Cancer J Clin 66: 271-289, 2016.

4. Kamangar F, Dores GM and Anderson WF: Patterns of cancer incidence, mortality, and prevalence across five continents: Defining priorities to reduce cancer disparities in different geographic regions of the world. J Clin Oncol 24: 2137-2150, 2006.

5. Thrumurthy SG, Chaudry MA, Chau I and Allum W: Does surgery have a role in managing incurable gastric cancer? Nat Rev Clin Oncol 12: 676-682, 2015.

6. Bartel DP: MicroRNAs: Genomics, biogenesis, mechanism, and function. Cell 116: 281-297, 2004.

7. Wang G, Fu Y, Liu G, Ye Y and Zhang X: miR-218 Inhibits Proliferation, Migration, and EMT of Gastric Cancer Cells by Targeting WASF3. Oncol Res 25: 355-364, 2017.

8. Lv H, Zhang Z, Wang Y, Li C, Gong W and Wang X: MicroRNA-92a promotes colorectal cancer cell growth and migration by inhibiting KLF4. Oncol Res 23: 283-290, 2016.

9. Ji S, Zhang B, Kong Y, Ma F and Hua Y: miR-326 inhibits gastric cancer cell growth through downregulating NOB1. Oncol Res 25: 853-861, 2017.

10. Liang J, Zhang Y, Jiang G, Liu Z, Xiang W, Chen X, Chen Z and Zhao J: MiR-138 induces renal carcinoma cell senescence by targeting EZH2 and is downregulated in human clear cell renal cell carcinoma. Oncol Res 21: 83-91, 2013.

11. He C, Yu T, Shi Y, Ma C, Yang W, Fang L, Sun M, Wu W, Xiao F, Guo F, et al: MicroRNA 301A promotes intestinal inflammation and colitis-associated cancer development by inhibiting BTG1. Gastroenterology 152: 1434-1448.e15, 2017.

12. Sun HL, Cui R, Zhou J, Teng KY, Hsiao YH, Nakanishi K, Fassan M, Luo Z, Shi G, Tili E, et al: ERK Activation globally downregulates miRNAs through phosphorylating exportin-5. cancer cell 30: 723-736, 2016.

13. Jin Y, Tao LP, Yao SC, Huang QK, Chen ZF, Sun YJ and Jin SQ: MicroRNA-582-5p suppressed gastric cancer cell proliferation via targeting AKT3. Eur Rev Med Pharmacol Sci 21: 5112-5120, 2017.

14. Qu Y, Zhang H, Sun W, Han Y, Li S, Qu Y, Ying G and Ba Y: MicroRNA-155 promotes gastric cancer growth and invasion by negatively regulating transforming growth factor- $\beta$ receptor 2 . Cancer Sci 109: 618-628, 2018

15. Guan H, Li W, Li Y, Wang J,Li Y, Tang Y and Lu S: MicroRNA-93 promotes proliferation and metastasis of gastric cancer via targeting TIMP2. PLoS One 12: e0189490, 2017.

16. Hwang SJ, Lee HW, Kim HR, Song HJ, Lee DH, Lee H, Shin CH, Joung JG, Kim DH, Joo KM, et al: Overexpression of microRNA-95-3p suppresses brain metastasis of lung adenocarcinoma through downregulation of cyclin D1. Oncotarget 6: 20434-20448, 2015.

17. Huang Z, Huang S, Wang Q, Liang L, Ni S, Wang L, Sheng W, He X and Du X: MicroRNA-95 promotes cell proliferation and targets sorting Nexin 1 in human colorectal carcinoma. Cancer Res 71: 2582-2589, 2011.

18. Fan B, Jiao BH, Fan FS, Lu SK, Song J, Guo CY, Yang JK and Yang L: Downregulation of miR-95-3p inhibits proliferation, and invasion promoting apoptosis of glioma cells by targeting CELF2. Int J Oncol 47: 1025-1033, 2015.

19. Chen J, Sun D, Chu H, Gong Z, Zhang C, Gong B, Li Y, Li N and Jiang L: Screening of differential microRNA expression in gastric signet ring cell carcinoma and gastric adenocarcinoma and target gene prediction. Oncol Rep 33: 2963-2971, 2015.

20. Jun KH, Lee JS, Kim JH, Kim JJ, Chin HM and Park SM: The rationality of $\mathrm{N} 3$ classification in the 7th edition of the International Union Against Cancer TNM staging system for gastric adenocarcinomas: A case-control study. Int J Surg 12: 893-896, 2014.

21. Livak KJ and Schmittgen TD: Analysis of relative gene expression data using real-time quantitative PCR and the 2(-Delta Delta C(T)) Method. Methods 25: 402-408, 2001. 
22. Chen $X$, Chen S, Hang W, Huang $H$ and Ma H: MiR-95 induces proliferation and chemo- or radioresistance through directly targeting sorting nexin1 (SNX1) in non-small cell lung cancer. Biomed Pharmacother 68: 589-595, 2014.

23. Xiao Z, Ching Chow S, Han Li C, Chun Tang S, Tsui SK, Lin Z and Chen Y: Role of microRNA-95 in the anticancer activity of Brucein D in hepatocellular carcinoma. Eur J Pharmacol 728: 141-150, 2014

24. Ng EK, Chong WW, Jin H, Lam EK, Shin VY, Yu J, Poon TC, Ng SS and Sung JJ: Differential expression of microRNAs in plasma of patients with colorectal cancer: A potential marker for colorectal cancer screening. Gut 58: 1375-1381, 2009.

25. Liu W, Li M, Chen X, Zhang D, Wei L, Zhang Z, Wang S, Meng L, Zhu S and Li B: MicroRNA-373 promotes migration and invasion in human esophageal squamous cell carcinoma by inhibiting TIMP3 expression. Am J Cancer Res 6: 1-14, 2015.
26. Yang F, Liu X, Liu Y, Liu Y, Zhang C, Wang Z, Jiang T and Wang Y: miR-181d/MALT1 regulatory axis attenuates mesenchymal phenotype through $\mathrm{NF}-\kappa \mathrm{B}$ pathways in glioblastoma. Cancer Lett 396: 1-9, 2017.

27. Gupta GP and Massagué J: Cancer metastasis: Building a framework. Cell 127: 679-695, 2006

28. Shishodia G, Shukla S, Srivastava Y, Masaldan S, Mehta S, Bhambhani S, Sharma S, Mehrotra R, Das BC and Bharti AC: Alterations in microRNAs miR-21 and let-7a correlate with aberrant STAT3 signaling and downstream effects during cervical carcinogenesis. Mol Cancer 14: 116, 2015. 